\title{
Adverse events associated with 2010 CSL and other inactivated influenza vaccines
}

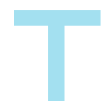
he 2010 trivalent influenza vaccine (TIV) manufactured in Australia by CSL Biotherapies (CSL) caused an excess of febrile reactions, including febrile convulsions, among Australian children. ${ }^{1}$ A retrospective cohort study conducted in Western Australia found that $57 \%$ of 209 children aged 6-59 months who received CSL TIV experienced a febrile reaction, compared with $17 \%$ of 110 children who received another TIV $(P<0.0001){ }^{1}{ }^{1}$ The Australian Technical Advisory Group on Immunisation concluded that the rate of febrile convulsions among children vaccinated with the 2010 CSL TIV may have been as high as 1 per $100 .^{2}$ The Therapeutic Goods Administration (TGA) stated that the cause of these reactions had still not been determined as of 8 July 2011. ${ }^{3}$

In this article, we consider a common factor in several clusters of adverse events following immunisation (AEFI) in the past 15 years - the use of deoxycholate as the virus-splitting agent in the manufacture of influenza vaccines.

\section{Split-virus and whole-virus vaccines}

In the 1960s, the introduction of a manufacturing process to chemically disrupt or "split" influenza viruses successfully reduced the reactogenicity of seasonal vaccines, generally without compromising immunogenicity. ${ }^{4}$ Inactivated (killed) virus, split through detergent or solvent solubilisation of the lipid membrane, still forms the basis for currently manufactured split influenza virus vaccines, including CSL influenza vaccines. ${ }^{4,5}$

Compared with whole-virus vaccines, split-virus vaccines have been shown to have a much improved profile for AEFI among all age groups. ${ }^{4}$ For example, a 1977 trial found that only $10 \%$ of 68 children who received a split-virus vaccine had rectal temperatures of $\geqslant 100^{\circ} \mathrm{F}$ compared with $40 \%$ of 65 children who received whole-virus vaccine $(P<0.01) .{ }^{6}$ Similarly, of 333 hospital staff being vaccinated for the first time and receiving a 1989 seasonal TIV, 13\% of those who received a split-virus formulation reported generalised aching during the 48 hours after vaccination, compared with $26 \%$ of those who received a whole-virus formulation $(P<0.01){ }^{7}$

Inactivation of the influenza virus is mainly achieved using $\beta$-propiolactone or formaldehyde. ${ }^{4} \mathrm{CSL}$ uses $\beta$-propiolactone to inactivate the virus but is one of only a few manufacturers globally to use deoxycholate (specifically, sodium taurodeoxycholate) as a splitting agent. $^{5}$

\section{Summary \\ - The 2010 trivalent influenza vaccine (TIV) manufactured by CSL Biotherapies (CSL) was associated with increased febrile reactions, including febrile convulsions, among Australian children. \\ - $\mathrm{CSL}$ is one of the few manufacturers that use deoxycholate as the virus-splitting agent in the manufacture of TIV. Clusters of adverse events following immunisation $(\mathrm{AEFI})$ have been previously linked to other deoxycholate-split TIV formulations in Europe and Canada. \\ - We hypothesise that suboptimal virus splitting or other mechanisms related to the use of deoxycholate may have played a role in adverse events linked to the 2010 CSL TIV. \\ - This hypothesis garners support from a recent United States Food and Drug Administration warning letter indicating that CSL failed to determine optimal splitting conditions for new virus strains and that assays to assess virus splitting had not been validated. \\ - While there may be other causes, the use of deoxycholate should be further explored. Comprehensive and timely investigations of AEFI, especially those involving children, are necessary to prevent their recurrence and to maintain public confidence in vaccination programs.}

BSC, MB BS, MPH, Head

Danuta M Skowronski MD, FRCPC, Lead, Influenza and Emerging Respiratory Pathogens $^{2}$

Gaston De Serres MD, PhD, Director Epidemiology ${ }^{3}$

Paul V Effler $\mathrm{MD}, \mathrm{MPH}$, Director. Prevention and Control Program ${ }^{4}$

1Epidemiology Unit Victorian Infectious Diseases Reference Laboratory, Melbourne, VIC.

$2 \mathrm{BC}$ Centre for Disease Control, Vancouver, British Columbia, Canada.

3 Institut national de santé publique du Québec, Québec, Canada.

4 Communicable Disease Control Directorate, WA Health, Perth, WA.

heath.kelly@mh.org.au

MJA 2011; 195: 318-320 doi: 10.5694/mjall.10941

Clinical focus p 336

\section{Deoxycholate-related clusters of adverse events following immunisation}

During the 2000-2001 influenza immunisation campaign in Canada, a novel adverse event designated oculorespiratory syndrome (ORS) - was identified in association with a domestically manufactured split-virus TIV. ${ }^{8}$ Although systemic symptoms were also reported, the syndrome was recognised because of allergic-like ocular and respiratory symptoms. ${ }^{8}$ Of 960 reported ORS cases, 96\% of the 937 cases where the administered vaccine brand was known followed receipt of a domestically produced deoxycholate-split TIV, while only $1 \%$ were reported following receipt of an imported TIV that used Triton X-100 (Sigma-Aldrich, St Louis, Mo, USA) as the splitting agent. ${ }^{9}$ The precise number of doses administered during the immunisation campaign was not available but, based on case reports and doses distributed, ORS was reported 150 times more frequently in association with the deoxycholate-split vaccine than with the Triton $X-100$ split vaccine. ${ }^{9}$

In a review of this cluster, the Global Advisory Committee on Vaccine Safety of the World Health Organization summarised that 
an investigation into the manufacturing process and transmission electron microscopy of the vaccine material found that there was variation in the process of disaggregation of virion particles, resulting in a disproportionate number of unsplit virion aggregates in the implicated product. ${ }^{10}$

This was thought to particularly apply to the A/ Panama/2007/99 H3N2 component that was introduced as a new vaccine strain in $2000-2001 .{ }^{11}$ With appropriate remedial action, including the supplementary use of Triton $\mathrm{X}-100$ as a second splitting agent, the problem was largely resolved the following season. ${ }^{9}$

At that time, ORS was thought to have first emerged in Canada. However, investigations revealed that a similar cluster of adverse events had occurred at least 5 years earlier in several European countries. ${ }^{8,12}$ The European cluster was associated with an influenza vaccine from another manufacturer that used deoxycholate as the splitting agent. Morphological aberrations similar to those identified in the Canadian vaccine were also reported with this vaccine. ${ }^{8}$ In Italy, the vaccine was associated with a 10-fold increase in AEFI reported during 1995-1996.8,12 In Czechoslovakia, the experience led to a call for systematic evaluation of AEFI that were possibly due to partially disrupted virions, as well as the routine morphological examination of individual vaccine lots as part of release control. ${ }^{13}$

Differential disruption of virions treated with various splitting agents was suspected as far back as 1984, when it was observed that

possibly the subunits produced by sodium deoxycholate are larger than those produced by other disruptive agents and their antigenic properties may, in consequence, approximate that of whole viruses. ${ }^{14}$

Based on earlier paediatric experience with wholevirus vaccines, inadequately split virions might be expected to trigger febrile reactions in young children, although the mechanism for this is not understood.

\section{Investigations into 2010 CSL trivalent influenza vaccine reactogenicity}

An interim report from the TGA published in October 2010 identified an increase in the neuraminidase concentration of the CSL 2010 seasonal vaccine as a possible cause for the reported AEFI. ${ }^{15}$ At the time, a number of other potential causes were excluded, including increased haemagglutinin concentration and the presence of live virus, endotoxins or contaminants. ${ }^{15}$ The report also included investigations to detect whole virus particles. None were detected, even when the final vaccine product was concentrated by ultracentrifugation. ${ }^{15}$ However, the extent to which incomplete splitting was ruled out appeared to be uncertain, with the report acknowledging that "a significant presence of intact viral particles would have been a concern with regard to potential pyrogenicity from whole virus particles". ${ }^{15}$

In the United States, CSL TIV was initially approved by the Food and Drug Administration (FDA) in 2007 for use in adults, and in 2009 for use in children aged 6 months and

older. ${ }^{16}$ On 15 June 2011, the FDA issued a warning letter to CSL. ${ }^{17}$ After reviewing the US regulator's findings, the Australian regulator (the TGA) concluded that, "The two regulators are in agreement over the problems identified at CSL". ${ }^{3}$ The FDA highlighted potential issues with the splitting procedure used by CSL. ${ }^{17}$ According to the FDA, sodium taurodeoxycholate lots that failed identification tests at CSL were nonetheless accepted for use. The FDA also stated that CSL "failed to determine optimal splitting conditions for new virus strains before the strains [were] used in production" and that "the tests used to evaluate the completeness of virus splitting [were] deficient" as the assays used were not "validated for their ability to discriminate between split and whole virus" ${ }^{17}$ These concerns, together with the previous Canadian and European experiences, suggest that incompletely split virus, and perhaps other factors related to the use of deoxycholate, provide a plausible explanation of the AEFI associated with the 2010 CSL TIV.

\section{Increased reactogenicity of CSL trivalent} influenza vaccine in children before 2010

the United States Food and Drug Administration

... issued a warning letter to CSL
While deoxycholate use in vaccine manufacturing was a common factor in several adverse event clusters between 1995 and 2001, it is important to note that no specific concerns have been raised about the safety profile of deoxycholate-split TIV products between 2001 and 2010. However, limited data from two clinical studies suggest that CSL TIV may have been, at least intermittently, associated with increased rates of febrile reactions in children before 2010 . The only paediatric trial of CSL TIV published to date reported that $22.5 \%$ of participants under 3 years of age experienced fever after receiving the 2005 formulation, but this figure rose to $39.5 \%$ after vaccination with the 2006 formulation $(P<0.005)$; one of the 272 study participants vaccinated in 2006 had a febrile convulsion. ${ }^{18}$ Furthermore, data emerging from a randomised trial conducted in the US in 2009 show that $37 \%$ of children aged 6 months to 3 years who received a first dose of 2009-2010 CSL TIV manufactured in Australia for the influenza season in North America experienced a febrile reaction, compared with $14 \%$ of those who received a first dose of a comparator brand of 2009-2010 TIV that was manufactured using Triton $X-100$ as the splitting agent $(P<0.0005){ }^{19,20}$

Implicating deoxycholate in increased reactogenicity associated with 2010 CSL TIV remains speculative. Its mechanism of action may not be limited to the effects of whole or incompletely split virus; the effect on the immune response of increased aggregation and altered antigenic presentation of surface proteins (including neuraminidase) or internal viral components may also be significant. Lipid remnants, and perhaps even residuals of the splitting agent itself, are less likely to be important. Should suboptimal virus splitting secondary to deoxycholate use ultimately be implicated as a factor in the increased reactogenicity of CSL TIV, the problem may be intermittent, and may potentially be solvable, as the deoxycholate parameters can be adjusted to ensure optimal disruption of specific virus strains. ${ }^{17}$ 


\section{Conclusion}

The benefits of TIV to those at risk of severe influenza complications should not be lost in this analysis. Acceptance of population-based vaccination programs depends on public trust in safe manufacturing, together with open and robust regulatory monitoring. Such trust is gradually earned but more easily eroded. A comprehensive and timely explanation of major episodes of AEFI, and the measures taken to prevent recurrences, is a reasonable public expectation.

Competing interests: Heath Kelly has received funding from CSL Biotherapies to attend a study design meeting. Danuta Skowronski was the principal investigator on a clinical trial for which influenza vaccine was provided free by Sanofi Pasteur. Gaston De Serres has previously received research grants for unrelated studies from GlaxoSmithKline and Sanofi Pasteur. The views expressed are ours and may not represent the views of our respective institutions.

Provenance: Not commissioned; externally peer reviewed.

1 Armstrong PK, Dowse GK, Effler PV, et al. Epidemiological study of severe febrile reactions in young children in Western Australia caused by a 2010 trivalent inactivated influenza vaccine. BMJ Open 2011; 30 May. doi:10.1136/ bmjopen-2010-000016.

2 Australian Technical Advisory Group on Immunisation. Clinical advice for immunisation providers on resumption of the use of 2010 trivalent seasonal vaccines in children less than 5 years of age, 30 July 2010. http:// www.health.gov.au/internet/immunise/Publishing.nsf/content/immuniseatagi-statement-tiv (accessed Aug 2011).

3 Therapeutic Goods Administration. The TGA and the USA FDA agree about problems at CSL Biotherapies. Canberra: TGA, 2011. http://www.tga.gov.au/ newsroom/btn-csl-tga-statement-110708.htm (accessed Aug 2011).

4 Furminger IGS. Vaccine production. In: Nicholson KG, Webster RG, Hay AJ, editors. Textbook of influenza. Oxford: Blackwell Science, 1998: 324-332.

5 Institute for Vaccine Safety. Excipients in vaccines. Baltimore: IVS, 2010. http:// hopkinsvaccine.org/components-Excipients.htm (accessed Aug 2011).

6 Gross PA, Ennis FA, Gaerlan PF, et al. A controlled double-blind comparison of reactogenicity, immunogenicity and protective efficacy of whole-virus and split-influenza product vaccines in children. J Infect Dis 1977; 136: 623-632.

7 al-Mazrou A, Scheifele DW, Soong T, Bjornson G. Comparison of adverse reactions to whole-virion and split-virion influenza vaccines in hospital personnel. CMAJ 1991; 145: 213-218.
8 Skowronski DM, Strauss B, De Serres G, et al. Oculo-respiratory syndrome: a new influenza vaccine-associated adverse event? Clin Infect Dis 2003; 36: 705713.

9 National Advisory Committee on Immunization. Supplementary statement for the 2001-2002 season: influenza vaccination of persons who experienced oculo-respiratory syndrome following previous influenza vaccination. Can Commun Dis Rep 2001; 27: 1-7.

10 Global Advisory Committee on Vaccine Safety. Oculorespiratory syndrome. Week Epi Rec 2003; 78: 17-24. http://www.who.int/wer/2003/en/ wer7804.pdf (accessed Jul 2011).

11 Skowronski DM, De Serres G, Scheifele D, et al. Randomized, double-blind, placebo-controlled trial to assess the rate of recurrence of oculorespiratory syndrome following influenza vaccination among persons previously affected. Clin Infect Dis 2003; 37: 1059-1066.

12 Splia-Alegani S, Salmaso S, Rota MC, et al. Reactogenicity in the elderly of nine commercial influenza vaccines: results from the Italian SVEVA study. Vaccine 1999; 17: 1898-1904.

13 Tumova B, Schramlova J, Vitkova E, et al. Is the release control of influenza vaccine sufficient? In: Programs and abstracts of Options for the Control of Influenza IIl; Cairns, Queensland; 1996.

14 Tannock GA, Bryce DA, Hensley MJ, et al. Responses to one or two doses of a deoxycholate subunit influenza vaccine in a primed population. Vaccine 1984; 2:100-106.

15 Therapeutic Goods Administration. Overview of vaccine regulation and safety monitoring and investigation into adverse events following 2010 seasonal influenza vaccination in young children. Attachment E: TGA influenza seasonal trivalent influenza vaccine laboratory investigation program. http:// www.tga.gov.au/safety/alerts-medicine-seasonal-flu101008.htm\#attachments (accessed Aug 2011).

16 Centers for Disease Control and Prevention (US). ACIP recommendation for use of CSL influenza vaccine [media statement]. 6 Aug 2010. http:// www.cdc.gov/media/pressrel/2010/s100806.htm (accessed Sep 2011).

17 Food and Drug Administration (US). Inspections, compliance, enforcement, and criminal investigations. Warning letter to Commonwealth Serum Laboratories (CSL) Ltd. June 15, 2011. http://www.fda.gov/ICECl/ EnforcementActions/WarningLetters/ucm259888.htm (accessed Jul 2011).

18 Nolan T, Richmond PC, McVernon J, et al. Safety and immunogenicity of an inactivated thimerosal-free influenza vaccine in infants and children. Influenza Other Resp Viruses 2009; 3: 315-325.

19 Commonwealth Serum Laboratories. Influenza virus vaccine, 2010-2011 formula (STN BL 125254/181). Highlights of prescribing information. http:// www.fda.gov/downloads/biologicsbloodvaccines/vaccines/ approvedproducts/ucm220730.pdf (accessed Aug 2011).

20 ClinicalTrials.gov. A study to determine the immunogenicity and safety profile of CSL Limited's influenza virus vaccine compared to a US licensed comparator influenza virus vaccine in a pediatric population. http://clinicaltrials.gov/ct2/ show/results/NCT00959049 (accessed Aug 2011). 